\title{
Two complexes that are spines of the three ball
}

by

\author{
Paul F. Dierker (Moscow, Idaho)
}

Let $M^{3}$ be a 3-manifold with 2-sphere boundary. Using the usual terminology, we will call $M^{3}$ a fake 3-ball if $M^{3}$ has the homotopy groups of a 3-ball, and a homology ball if $M^{3}$ has the homology of a 3 -ball. It is well known that there are homology 3-balls that are not 3-balls while the 3-dimensional Poincaré Conjecture (every fake 3 -ball is a 3-ball) is unsolved.

A 3-manifold with non-empty boundary can be collapsed to a 2-complex $K$ in the interior of the manifold. Moreover, we may assume, without loss of generality, that $K$ is a normalized spine of the 3-manifold. That is, every one simplex in $K$ is a face of at most three two simplexes [2]. In this paper we develop a criterion on a normalized spine of a homology 3 -ball that will ensure that the homology 3 -ball is in fact a 3 -ball.

Let $K$ denote a finite geometric $n$-complex. The following notation will be used.

$$
\begin{aligned}
& F(K)=\left\{\sigma^{n-1} \mid \sigma^{n-1} \text { is a face of exactly one } \sigma^{n} \in K\right\}, \\
& S(K)=\left\{\sigma^{n-1} \mid \sigma^{n-1} \text { is a face of more than two } \sigma^{n} \in K\right\} .
\end{aligned}
$$

That is, $F(K)$ is the union of all $(n-1)$-dimensional free faces of $K$, while $S(K)$ is the set of singular points of $K$.

Then we have:

Lemara 1. If $L=S(K) \cup F(K)$, then either $H_{n}(K, L)$ is non-trivial or $H_{n-1}(K, L)$ has a subgroup of order two.

Proof. Suppose that $H_{n}(K, L)$ is trivial and let $\left\{\sigma_{i}^{n} \mid i=1, \ldots, q\right\}$ be the set of all $n$-simplexes in $K$. Using the fact that every $(n-1)$ - simplex in $K-L$ is a face of precisely two $n$-simplexes, an easy calculation will show that if $\partial\left(\sum_{1}^{\alpha} \sigma_{i}^{n}\right)=\sum_{i \in I} 2 \alpha_{i} \sigma_{i}^{n-1}$ then $\sum_{i \in T} \alpha_{i} \sigma_{i}^{n-1}$ is in fact a non-trivial element of order two in $H_{n-1}(K, L)$. We will call a connected complex $K$ homologically trivial if $H_{q}(K)=0$ for all $q \geqslant 1$.

LEMMa 2. If $K$ is a homologically trivial $n$-complex with $n \geqslant 2$ and $L=S(K) \cup F^{\prime}(K)$, then either $H_{n-1}(L)$ is non-trivial or $H_{n-2}(L)$ has a subgroup of order two. 
Proof. From the augmented homology sequence of the pair $(K, L)$ and the hypothesis we find that $H_{q}(K, L)=H_{q-1}(L)$ for $q \geqslant 1$. The previous lemma then yields the desired result.

Note that in case $n=2$ in the above lemma $H_{1}(L)$ must be nontrivial.

LEMCMA 3. If $K$ is a homologically trivial connected 2-complex with $S(K)$ homologically trivial, then $K \searrow 0$.

Proof. From the previous lemma we must have $F(K) \neq \emptyset$. Thus we may start collapsing $K$. Suppose that $K \backslash K_{n}$ and we here reach an impass in our collapsing. That is, $K_{n}$ is a 2 -complex with no free faces. Clearly $S\left(K_{n}\right) \subset S(K)$, so $S\left(K_{n}\right)$ is homologically trivial, and from the previous lemma $F\left(K_{n}\right) \neq \emptyset$, a contradiction. Thus $K \backslash 0$.

Let $D_{n}$ be the polyhedron which is $n$ disjoint copies of $S^{1}$ joined by line segments as in Figure 1.
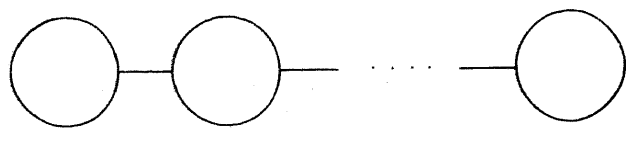

Fig. 1

Further, let $D$ be any polyhedron which is a disjoint union of copies of various $D_{n}$ 's. If $K$ is a two-complex, let $S_{1}(K)$ denote the set of all $x$ in $S(K)$ with a neighborhood which is the product of an are and a triode, and let $S_{2}(K)$ denote the set of all $x$ in $S(K)$ with a neighborhood which is a cone over $D$.

With this notation the following theorem may be given.

THEOREM 1. Let $K$ be a 2-complex which is the spine of a homology three cell $M^{3}$. If for each simple ctosed curve $C$ in $\mathbb{S}(K), C \cap \mathbb{S}_{2}(K) \neq \varnothing$, then $M^{3}$ is a 3-ball.

Proof. The idea is to expand $K$ to a complex $L \subset M^{3}$. Then since $M \searrow K$ and $L \searrow K$, we have by [2] that $M \searrow L$. $L$ will have been constructed in such a way that $L \searrow 0$. Thus $M \searrow 0$, and by [2] we have the desired result.

First expand $K$ at each point of $S_{2}(K)$ by introducing a 3-ball into each cone as indicated in Figure 2. The 3-complex so obtained is $L$.

It is clear that $L \backslash \boldsymbol{K}$. We now show $L \searrow 0$. First we collapse $L$ to two complex $L^{\prime}$ by collapsing out all the copies of $B^{3}$ introduced in the expansion of $K$ to $L$. The result of such collapses is indicated for a typical case in Figure 3.

Note that these operations introduce no new singularities so that $\boldsymbol{S}\left(\boldsymbol{L}^{\prime}\right) \boldsymbol{C} \boldsymbol{S}(\boldsymbol{K})$. Moreover, note that by hypothesis these operations remove a 1-cell from every simple closed curve in $S(K)$. Thus $S\left(L^{\prime}\right)$ is homotopically trivial.

Since expansions and collapses are homotopy equivalences, and $M^{3}$ was homologically trivial, $L^{\prime}$ is homologically trivial. Thus by Lemma 3 , $L^{\prime} \searrow 0$ and the proof is complete.

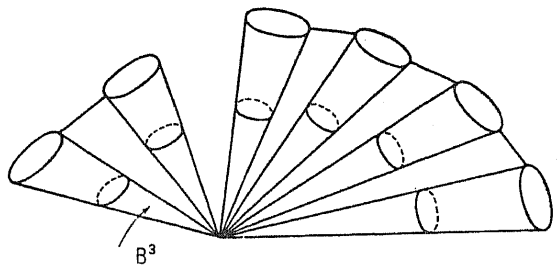

Fig. 2

It is clear that the proof of the previous theorem depends only on "breaking" the simple closed curves in $S(K)$. This theorem was not intended to be exhaustive. For example, the situation may arise where

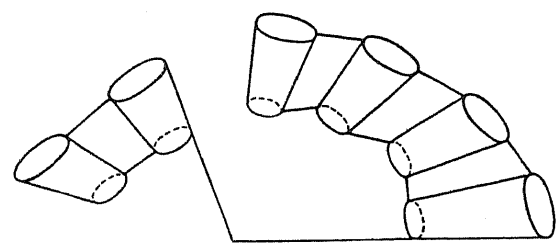

Fig. 3

a simple closed curve in $S(K)$ is embedded in $K$ as indicated in Figure 4 (consider the house with two rooms for example). This simple closed curve may be broken by "fattening" the disk $D$ to a 3-ball and collapsing the 3 -ball across the 2 -ball $B^{2}$ as indicated in Figure 5 .

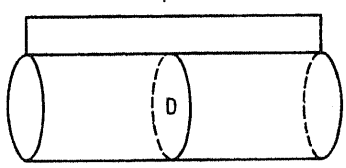

Fig. 4

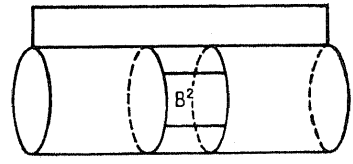

Fig. 5
Perhaps a modification of the method used by Casler in [1] could be used to establish the following:

Oonjecture. Every connected 3-manifold with non-empty boundary has a spine $K$ for which $S(K)=S_{1}(K) \cup S_{2}(K)$. 


\section{References}

[1] B. G. Casler, An imbedding theorem for connected 3-manifolds with boundary, Proc. Amer. Math. Soc. 16 (1965), pp. 559-566.

[2] E. C. Zeeman, Seminar on Combinatorial Topology (mimeographed notes), Institute des Hautes Etudes Scientifiques, Paris 1963.

\section{UNIVERSITY OF IDAHO}

Moscow, Idaho

\section{Measurable cardinals and analytic games}

by

\section{Donald A. Martin (New York)}

Introduction. A subset $P$ of $\omega^{\omega}$ is determinate if, in the sense of [5] the game $G_{\omega}(P)$ is determined. The assumption that every projective set is determinate implies that every projective set is Lebesgue measurable (see [6]) and leads to a complete solution to the problem of reduction and separation principles in the classical and effective projective hierarchies [1], [4]. Because if these and other consequences it would be pleasant to have a proof that every projective set is determinate. The best available result is that every $\boldsymbol{F}_{\sigma \delta}$ is determinate [2]. It is not provable in Zermelo-Fraenkel set theory that every analytic $\left(\mathbf{s}_{1}^{1}\right)$ set is determinate [5]. $\left({ }^{1}\right)$

We assume the existence of a measurable cardinal and prove that every analytic set is determinate. Oux proof is fairly simple and makes a very direct use of the large cardinal assumption (we present it in terms of a Ramsey cardinal) and the fact that open games are determined. We believe that larger cardinals will yield a generalization of our proof to all projective sets. The assumption that measurable cardinals exist is known not to imply even that all $\boldsymbol{A}_{2}^{1}$ sets are determinate. (This follows from [1], [4] and work of Silver.)

\$1. Definitions. (For more information on the analytical hierarchy see [7], [8]; on infinite games see [5]; on large cardinals see [10], [11].)

Lét $\omega$ be the set of all natural numbers. If $f: \omega \rightarrow A$, the function $\bar{f}$ is defined by setting $\bar{f}(n)$ equal to the sequence $\langle f(0), f(1), \ldots, f(n-1)\rangle$ of the first $n$ values of $f$. Let Seq be the set of all finite sequences of natural numbers. Let $n \rightarrow k_{n}$ be some enumeration of Seq with the property that $k_{n}$ has length $\leqslant n$. The Kleene-Brouwer ordering of Seq is defined by

$$
\bar{f}(m)<\bar{g}(n) \leftrightarrow\left\{\begin{array}{l}
\bar{f}(m) \text { is a proper extension of } \bar{g}(n), \\
\text { or at the least } p \text { for which } f(p) \neq g(p), \\
f(p)<g(p) .
\end{array}\right.
$$

(1) Harvey Friedman (unpublished) has shown that the determinateness of Borel sets cannot be proved in Zermelo set theory. Whether it can be proved in Zermelo-Fraenkel set theory remains open. 